Editorial

\title{
The Interventional Radiology Residency: Is It Time Saudi Radiology Residency Programs Contemplate the Idea?
}

\author{
Abdullah Abohimed $^{10}$ Yousof Al Zahrani ${ }^{2}$ Mohammad Arabi ${ }^{1}$ \\ ${ }^{1}$ King Abdulaziz Medical City \& King Abdullah Specialist Hospital, \\ Ministry of National Guard Health Affairs, Riyadh, Kingdom of Saudi \\ Arabia \\ 2 Vascular and Interventional Radiology Unit, King Abdulaziz Medical \\ City \& King Abdullah Specialist Hospital, Ministry of National Guard \\ Health Affairs, Kingdom of Saudi Arabia
}

Arab J Intervent Radiol 2021;5:57-59.

It would be an understatement to say that interventional radiology (IR) is advancing at an exponential rate. The minimally invasive nature of procedures provided by IR has proven to be cost-effective, favored by patients, and an excellent alternative to conventional surgery. This has led to higher demand for the services provided by IR by many medical specialties. Despite this, ambiguity and confusion still loom large when it comes to the perception and awareness of IR among medical faculty and medical students, respectively. This could be attributed to many factors. ${ }^{1,2}$

In Saudi Arabia, the inaugural IR fellowship training program had begun in the year 2000. It was structured as a 1-year fellowship program after the completion of a 4-year Diagnostic Radiology (DR) residency. Four years later, the Saudi Commission for Health Specialties (SCFHS) had approved a 2-year fellowship program for IR after the prior single year program was met with much criticism. ${ }^{3}$ Today, there are a total of 8 IR fellowship training centers in the Kingdom and 24 fellows practicing IR. Other neighboring countries such as Egypt ${ }^{4}$ also offer a 2-year IR fellowship program. IR has yet to achieve specialty independence in the Kingdom. In a recent study, Makris et.al had found considerable heterogeneity both in exposure during IR training and training satisfaction on a global scale. ${ }^{5}$

The United States no longer offers a classic IR fellowship post DR residency. Instead, there exists three separate pathways to becoming an IRs in the United States. ${ }^{6}$ The "integrated" IR residency is a 5-year program that includes 3 initial years of DR followed by 2 years of IR. The "independent" IR residency is a 2-year residency that is independent and taken after the DR residency. The independent pathway also has its own matching process after the completion of a DR residency. The third pathway is known as "Early Specialization in IR" (ESIR). Here, DR residents who develop an early desire to specialize in IR can join the ESIR program. The ESIR resident would naturally have a different residency structure to his DR peers. The minimum requirements to graduate as an ESIR resident are 12 IR or IR-related rotations during the postgraduate year (PGY) 2-5; and one intensive care unit rotation. During said rotations, the ESIR resident must complete a minimum of 500 procedures and is required to maintain a logbook of such cases. ESIR residents are then eligible to match for the second year of the independent residency, lowering the number of years in training to 6 .

An extraordinary amount of effort was put in place when considering this drastic shift in training. ${ }^{7}$ Ultimately, the rationale behind it stems from a few important factors: First, although DR and IR share similar competencies in imaging and procedures, postprocedural patient care is a unique competency to IR. Second, the complexity of procedures offered by IR is increasing, necessitating more years of training. And third, more focused training in IR will prove beneficial to patients and patient care in the long run.

Current DR residency structure in Saudi Arabia permits a minimum of three and a maximum of six IR rotations during PGY-2-5. ${ }^{8}$ This consists of three mandatory "core" IR rotations taken during clinical years, and three nonmandatory "elective" rotations, from which a resident can choose IR. Some DR residencies in Saudi Arabia reserve one elective rotation for the American Institute for Radiologic Pathology course. This is in stark contrast to the United States, where the pivotal PGY-5 year is, on average, composed of nine elective rotations alone.
Address for correspondence Abdullah Abohimed, MBBS, Radiology Resident. King

Abdulaziz Medical City \& King Abdullah Specialist Hospital. Ministry of National Guard Health Affairs, P.O. Box 22490. Riyadh.

11426., Kingdom of Saudi Arabia, Mail Code 1904

(e-mail: AbdullahAbohimed@gmail. com).
DOI https://doi.org/ 10.1055/s-0041-1742087. ISSN 2542-7075. (c) 2022. The Pan Arab Interventional Radiology Society. All rights reserved.

This is an open access article published by Thieme under the terms of the Creative Commons Attribution-NonDerivative-NonCommercial-License, permitting copying and reproduction so long as the original work is given appropriate credit. Contents may not be used for commercial purposes, or adapted, remixed, transformed or built upon. (https://creativecommons.org/ licenses/by-nc-nd/4.0/)

Thieme Medical and Scientific Publishers Pvt. Ltd., A-12, 2nd Floor, Sector 2, Noida-201301 UP, India 
This means that the DR resident hoping to pursue the independent pathway would have received ample exposure and training in IR. However, the Saudi DR resident hoping to pursue an IR fellowship must rely on three core rotations and possibly a few more for electives.

There are a few inherent problems to the current Saudi DR structure: The IR fellow is expected to begin performing minor procedures and closely aid major procedures within the first few days of fellowship; this would require a more robust exposure during residency. Also, not all residents with an interest for IR eventually end up pursuing IR. Therefore, three core rotations may not be enough to tip the scale for the undecided resident.

IR fellowship and DR residency program directors, key stakeholders, and administrative officials must engage in serious dialogue about the current DR residency and IR fellowship structures. ${ }^{9}$ The importance of implementing an IR residency, or a similar program to that of ESIR, cannot be overstated. Advantages to a shift in training are many: Not only will you potentially produce more competently trained IRs, the IR or ESIR resident can contribute to IR calls, clinic duties, IR consults, and the imaging workflow.

We ran a pilot cross-sectional, survey-based study involving 25 current Saudi IR fellows and IRs who have completed their fellowship training within 3 to 12 months (-Fig. 1) across the Riyadh, Jeddah, Makkah, and Dammam regions (see Appendix) Almost all respondents (92\%) agree with implementing a different training pathway for IR similar to that of ESIR or the integrated pathway (-Fig. 2). Sixty percent believe three core IR rotations during DR residency are not enough to supplement the trainee with the needed foundation to function well during the first few months of fellowship (-Fig. 3). Similarly, the majority had found there first few months of fellowship to be extremely difficult (-Fig. 4) Some of the reasons included were: "I knew very little about the equipment used in IR" (62.5\%); "I was not aware of the pre-procedural side of IR" (41.6\%); "I was not aware of the indications and contraindications to many IR procedures" (37.5\%); and "I needed time to adjust at my new training center" (20.83\%).

IR has always been at the forefront of procedural innovation, and to ensure a constant stream of passionate

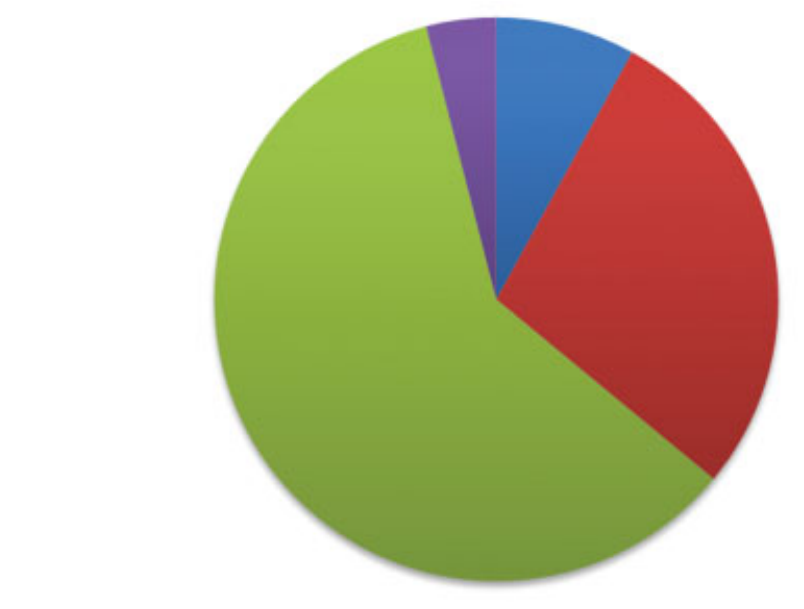

w 1st year of fellowship (F1)

a 2nd year of fellowship (F2)

घecently completed my fellowship training $\mathbf{a}$ Other (please specify)

Fig. 1 Current level of survey responders.

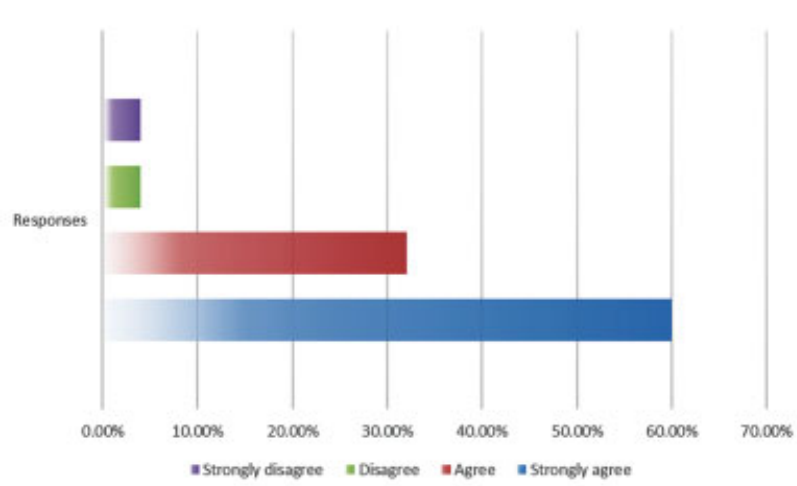

Fig. 2 Do you think it is time the Saudi Commission for Health Sciences introduces a Saudi integrated interventional radiology (IR) residency or Early Specialization in IR?

and enthusiastic medical trainees pursuing careers in IR, it is imperative that this subject is discussed thoroughly during the next annual Saudi Interventional Radiology Society (SIRS) and Pan Arab Interventional Radiology Society (PAIRS) conferences. Furthermore, dedicated

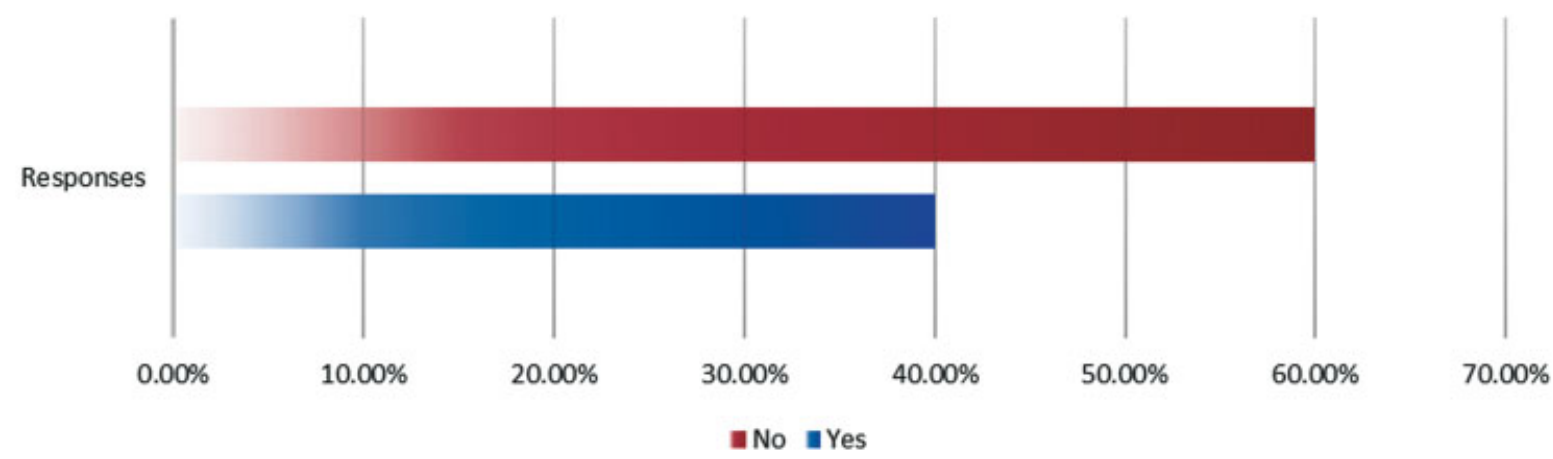

Fig. 3 Do you think three core interventional radiology (IR) rotations during the 3rd and 4th years of residency are enough to give a new IR fellow the needed foundation and fundamentals to function well during the first few months of fellowship training? 


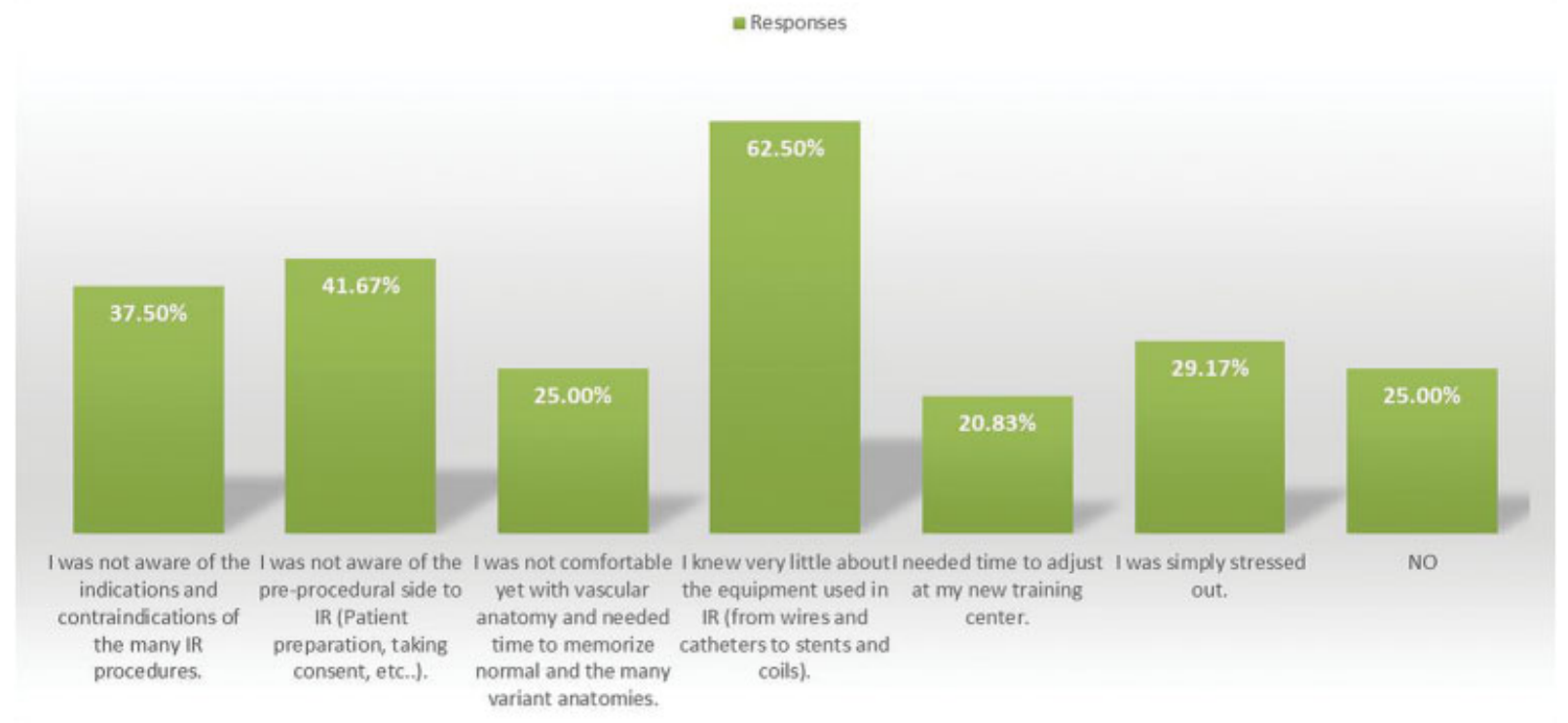

Fig. 4 Have the first few months of interventional radiology (IR) fellowship training been difficult for you?

medical student IR conferences sponsored by the SIRS with an emphasis on spreading awareness of IR should become a priority. The PAIRS has made inroads in this department with the success of its IR awareness campaign titled \#I_AM_PAIRS, and the formation of a resident, student, and fellow committee.

\section{Conflict of Interest}

None.

\section{Acknowledgment}

None.

\section{References}

1 Doherty MG. Value of interventional radiology: past, present, and future. Semin Intervent Radiol 2019;36(01):26-28
2 Abohimed AB, Al Zahrani Y, Arabi M. Interventional radiology awareness among the final-year medical students in Riyadh. Arab J Intervent Radiol 2020;4:32-37

3 Al Zahrani YA, Arabi M, Almoaiqel M. The status of vascular and interventional radiology training: the Saudi experience. Arab J Intervent Radiol 2019;3:42-43

4 El Tawab KA, Wahab SM. The status of interventional radiology training: the Egyptian experience. Arab J Intervent Radiol 2020;4:3

5 Makris GC, Burrows V, Lyall F, Moore A, Hamady MS. Vascular and interventional radiology training; international perspectives and challenges. Cardiovasc Intervent Radiol 2021;44(03):462-472

6 Keefe N, Doster J. The new IR residency is here: is your practice ready? Appl Radiol 2021;50(02):16-18

7 Kaufman JA. The interventional radiology/diagnostic radiology certificate and interventional radiology residency. Radiology 2014;273(02):318-321

8 Saudi Commission for Health Specialties. Saudi Board Medical Imaging Curriculum. Saudi Commission for Health Specialties; 2015. Accessed December 20, 2021 from: http://www.scfhs.org.sa 9 Marx MV, Sabri SS. Interventional radiology residency: steps to implementation. J Am Coll Radiol 2015;12(08):854-859 